\title{
Measurement of torsional rigidity of yarns with different crimps
}

\author{
Julie Peiffer ${ }^{1}$, KyoungOk Kim², Masayuki Takatera ${ }^{2}$
}

${ }^{1}$ Department of Bioscience and Textile Technology, Interdisciplinary Division of Science and Technology, Shinshu University, 3-15-1 Tokida, Ueda, Nagano 386-8567, Japan.

${ }^{2}$ Division of Kansei and Fashion Engineering, Institute for Fiber Engineering (IFES), Interdisciplinary Cluster for Cutting Edge Research (ICCER), Shinshu University, 3-15-1 Tokida, Ueda, Nagano 386-8567, Japan

\author{
takatera@shinshu-u.ac.jp
}

\begin{abstract}
The effect of crimp on torsional rigidity of monofilament and cotton spun yarns was studied. Two kinds of polymeric monofilament yarns and four kinds of cotton spun yarns were examined. Different crimps were applied to the yarn using original crimp setting equipment. To fix the crimp, the polymeric monofilaments were treated with heat and the cotton spun yarns were treated with steam. The test samples were then produced following two protocols: with or without the application of weight. The yarn torsional rigidities with crimp were measured using a torsional measurement device and were compared with those without crimp. Almost no weight was applied to the cotton spun yarns to preserve the crimp during testing. The results with and without the application of weight were compared. For the monofilament yarns, the torsional rigidities of the crimped yarns had a linear relationship with the crimp ratio. For the cotton spun yarns, the torsional rigidities of the crimped yarns were smaller than those of the straight yarns. The smaller the yarn count, the smaller the yarn torsional rigidity. The effect of crimp on torsional rigidity differed according to the yarn counts. There was almost no difference in crimped yarn torsional rigidity between the straightened and non-straightened yarns after crimp setting. Therefore, there is a possibility that the change in yarn properties could have resulted from the bending of the fiber during crimp setting and not from the shape of the crimp afterwards.
\end{abstract}

Keywords: crimp, torsional rigidity, torque, spun yarn, filament yarn

\section{Introduction}

When simulating the draping and bending behavior of a fabric, the fabric material, its structure, and the properties of the yarn must be taken into account. The bending, torsional, and tensile properties of the yarn are important for predicting the mechanical properties of the fabric [1-3].

The torsional properties of yarns have been widely studied over the years. Yarn properties have been investigated because they influence fabric bending properties and therefore the hand of the fabric [4-5].

Fiber crimp has been extensively studied for analyzing the geometrical structure and mechanical properties of fiber and yarn [6-8]. Meredith first developed a method to measure fiber crimp [9]. Subsequently, models of the geometry of fiber crimp were investigated [10]. Starting with Pierce's model, many studies have been carried out on yarn crimp geometry and its effect within the fabric [11-12]. However, only a few studies have been conducted on the mechanical properties of crimped yarn.

In 1958, Platt et al. [5] developed a model for the yarn torque of single yarns, taking into account fiber bending, fiber torsion, and a combination of the two. This study was continued by Postle et al. [13-14] who developed a model to estimate the torque for newly twisted as well as for continuous filament yarns.

To predict fabric bending properties it is necessary to estimate the effect of crimp on the torsional rigidity of the yarn. Cooper demonstrated the influence of yarn torsional rigidity on fabric torsional rigidity by establishing a model that included both the torsional and bending rigidity of the yarn [4]. Shinohara later pursued this research on the yarn torsional rigidity term of Cooper's model [15]. Peiffer et al. [16] showed that fabric bending properties could be predicted using Cooper's [4] and Shinohara et al.'s [15] models taking into account the torsional rigidity of the yarn to predict fabric bending rigidity. The study also showed an important difference between yarn torsional rigidity measured from the bobbin, and that measured when extracted from the fabric. The difference in properties between straight and crimped yarns could explain this discrepancy. 
Regarding the effect of crimp on yarn properties, Skelton [17] published an extensive study on the tensile, flexural, and torsional properties of crimped filaments. He made theoretical models of saw tooth, rectangular, and circular-arc crimps, and verified his theory by measuring the torsional rigidity of circular-arc piano wire and nylon monofilament yarn extracted from a fabric using a torsional pendulum. However, the torque-twist curve was not obtained due to the measuring method used. Furthermore, this study was not conducted for common spun yarn with undulating crimp. Ordinarily, experimental yarn with crimp extracted from a fabric is used for experiments. However, this does not allow much freedom in the choice of parameters, such as the wavelength or amplitude of the crimp. Furthermore, it would be more convenient for simulation purposes if it were possible to estimate the torsional rigidity of the yarn without weaving. The design of a torsional test for crimped spun yarn is problematic because of the difficulty in creating and maintaining crimp experimentally.

In this study, crimped monofilaments and spun yarns samples with various amplitudes and wavelengths were made using original crimp setting equipment. Then, the torque-twist curves of straight and crimped yarns were obtained using a torsional tester and determined their torsional rigidities. The effect of crimp on yarn torsional rigidity was investigated by comparing the torsional rigidities of crimped and straight yarns.

\section{Theoretical}

The theory of the torsional properties of crimped fibers has been discussed by Skelton [17]. If the bending rigidity of the straight filament is $B$, the torsional rigidity of the straight filament is $W$, and the torsional rigidity of the crimped filament is $W_{2}$, the relationship between $W$ and $W_{2}$ in the initial state is given by Equation (1):

$$
\frac{L}{W_{2}}=\frac{l}{W}+\left(\frac{1}{B}-\frac{1}{W}\right) \int_{0}^{l} \sin ^{2} f(s) \mathrm{d} s
$$

where $L$ is the projected length of the filament on the torsional axis, $l$ is the length of the filament along the crimp, and $\phi=f(s)$ is the configuration of the filament in a plane, given by $s$, the distance along the filament between the origin and $\phi$ the tangent angle at $s$. Skelton solved Equation (1) for circular crimp and produced Equation (2):

$$
\frac{1}{W_{2}}=(1+c)\left[\frac{1}{W}+\left(\frac{1}{B}-\frac{1}{W}\right)\left(\frac{1}{2}-\frac{\sin 2 \phi_{0}}{4 \phi_{0}}\right)\right]
$$

where $c$ is the crimp ratio and $\phi_{0}$ is the value of $\phi$ at $s=0$. In this Equation, $c$ and $\phi_{0}$ are linked by Equation (3):

Therefore,

$$
\frac{\phi_{0}}{\sin \phi_{0}}=1+c
$$

$$
\frac{W}{W_{2}}=(1+c)\left[\frac{1}{2}+\frac{\sin 2 \phi_{0}}{4 \phi_{0}}+\frac{W}{B}\left(\frac{1}{2}-\frac{\sin 2 \phi_{0}}{4 \phi_{0}}\right)\right]
$$

These equations describe the important relationship between crimp ratio and filament rigidity in the initial state. For the same crimp configuration yarn, the ratio $W / W_{2}$, which will be named afterward 'torsional rigidity ratio', is affected mainly by the bending rigidity $B$. However, the torque-twist relationship during twisting is not given because $\phi$ can no longer be considered a constant, and the configuration is no longer in a plane but rather in three dimensions.

\section{Experimental}

To investigate the effect of crimp on yarn torsional properties, different crimps were applied to monofilaments and cotton spun yarns. The torsional rigidity of the yarn was then measured and the results were compared.

\section{Crimp condition setting}

To investigate the effect of crimp on yarn torsional rigidity, we generated yarns with crimps of various amplitudes and wavelengths. Four types of crimp setting equipment were created using a box with many parallel metal rods with circular cross sections, as shown in Figure 1. The diameters $d_{r}$ of the rods were $\varnothing 1.2 \mathrm{~mm}, \varnothing 2 \mathrm{~mm}, \varnothing 3 \mathrm{~mm}$, and $\emptyset 4 \mathrm{~mm}$, and the interval $l$ between the rods was $d_{r}+1.5 \mathrm{~mm}$. Each yarn interlaced the rod as shown in Figure 1. Figure 2 shows the yarn geometry set-in for the equipment, with $d_{f}$ the diameter of the yarn, $\lambda$ the wavelength of the crimp, $A$ the amplitude of the crimp, and $s$ the yarn length for a wavelength $\lambda$ and an amplitude $A$. 


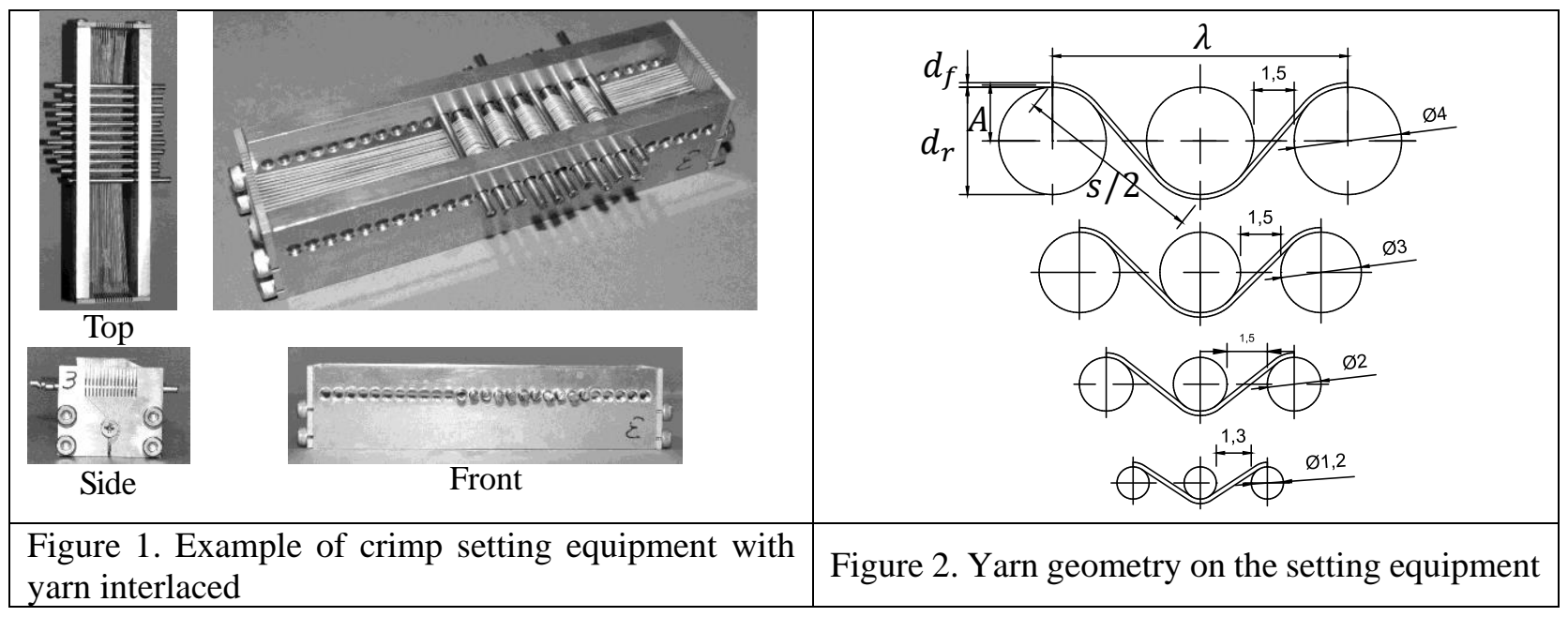

Setting of crimp for monofilament yarns. First, the monofilaments were set in position in the crimp setting equipment. To fix the crimp, the equipment was put in an oven at $160^{\circ} \mathrm{C}$ for $15 \mathrm{~min}$. Once the equipment had cooled to room temperature, the metal rods were removed leaving the crimped samples.

Setting of crimp for cotton spun yarns. To fix the crimp in the cotton spun yarns, the yarns were set in the equipment and subjected to steam treatment for 30 min using a common steam cooker. The samples were then left to dry in a thermohygrostat set to standard conditions $\left(20 \pm 1^{\circ} \mathrm{C}\right.$ and relative humidity of $\left.65 \pm 5 \%\right)$ for $24 \mathrm{~h}$.

\section{Samples}

Six types of yarn were used, as shown in Table 1. Nylon yarn (no.1, Toho Co., Ltd., Hiroshima, Japan) and fluorocarbon yarn (Basic FC, no. 1, Sunline Co., Ltd., Iwakuni, Japan) were used as monofilament yarns. The cotton yarn samples were made by ring spinning using the same combed cotton roving. The average diameter of cotton spun yarn was obtained using a digital micrometer (Keyence Corporation, LS7000, measurement accuracy $\pm 0.5 \mu \mathrm{m}$ ) at an angle from $0^{\circ}$ to $180^{\circ}$ [18].

Table 1. Yarn specifications

\begin{tabular}{|c|c|c|c|c|c|c|}
\hline Sample & Material & Structure & $\begin{array}{l}\text { Yarn count } \\
\text { (tex) }\end{array}$ & Twist factor & Twist (tpm) & $\begin{array}{l}\text { Diameter } \\
(\mathrm{mm})\end{array} d_{f}$ \\
\hline $\mathrm{FC}$ & Fluorocarbon & \multirow{2}{*}{ monofilament } & 45 & \multirow{2}{*}{-} & \multirow{2}{*}{ - } & 0.165 \\
\hline Nylon & Polyamide 6.6 & & 26 & & & 0.170 \\
\hline A & \multirow{4}{*}{$\begin{array}{l}\text { Combed } \\
\text { cotton }\end{array}$} & \multirow{4}{*}{ spun yarn } & 15 & \multirow{4}{*}{5.0} & 1234 & 0.156 \\
\hline $\mathrm{B}$ & & & 20 & & 1069 & 0.191 \\
\hline $\mathrm{C}$ & & & 30 & & 874 & 0.228 \\
\hline $\mathrm{D}$ & & & 40 & & 755 & 0.265 \\
\hline
\end{tabular}

The yarn setting conditions are shown in Table 2. The suffix refers to the diameter of the rods. The crimp $c$ was calculated using Equation (5), as defined by Pierce [11]:

$$
c=\frac{s}{\lambda}-1
$$

Table 2. Crimped yarn geometry at setting $(\mathrm{mm})$

\begin{tabular}{|l|llll|lllll|lllll|llll|}
\hline$d_{r}$ & $\varnothing 1.2$ & & & & $\varnothing 2$ & & & & $\varnothing 3$ & & & $\varnothing 4$ & & \\
\hline Samples & $A_{1.2}$ & $\lambda_{1.2}$ & $s_{1.2}$ & $c_{1.2}$ & $A_{2}$ & $\lambda_{2}$ & $s_{2}$ & $c_{2}$ & $A_{3}$ & $\lambda_{3}$ & $s_{3}$ & $c_{3}$ & $A_{4}$ & $\lambda_{4}$ & $s_{4}$ & $c_{4}$ \\
\hline FC & 1.28 & 5.00 & 7.55 & 0.51 & 2.08 & 7.00 & 11.53 & 0.65 & 3.08 & 9.00 & 16.60 & 0.84 & 4.08 & 11.00 & 21.88 & 0.99 \\
Nylon & 1.29 & 5.00 & 7.58 & 0.52 & 2.09 & 7.00 & 11.56 & 0.65 & 3.09 & 9.00 & 16.64 & 0.85 & 4.09 & 11.00 & 21.92 & 0.99 \\
\hline A & 1.30 & 5.00 & 7.50 & 0.50 & 2.08 & 7.00 & 11.48 & 0.64 & 3.08 & 9.00 & 16.53 & 0.84 & 4.08 & 11.00 & 21.81 & 0.98 \\
B & 1.31 & 5.00 & 7.68 & 0.54 & 2.10 & 7.00 & 11.69 & 0.67 & 3.10 & 9.00 & 16.79 & 0.87 & 4.10 & 11.00 & 22.10 & 1.01 \\
C & 1.31 & 5.00 & 7.88 & 0.58 & 2.11 & 7.00 & 11.92 & 0.70 & 3.11 & 9.00 & 17.07 & 0.90 & 4.11 & 11.00 & 22.42 & 1.04
\end{tabular}




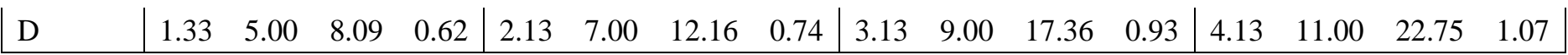

\section{Measurement of yarn torsional rigidity}

A Kawabata Evaluation System yarn torsional tester (KES)-YN1 (Kato Tech Co. Ltd., Kyoto, Japan) was used to obtain the torsional rigidity [16]. This device gives a measurement of the torque and the twist angle by measuring the resistance of the sample when rotating one extremity while the other is fixed. The movable part of the apparatus then makes a $6 \pi$ rotation in one direction followed by a $12 \pi$ rotation in the other, and returns to the original position while the device registers the torque and twist angle. Torsional rigidity is obtained from the mean of the two slopes between $2 \pi$ and $4 \pi$ in the forward rotation, and $-2 \pi$ and $-4 \pi$ in the backward rotation. The torsional rigidity $W_{\text {mes }}$ is then calculated using sample length $L$ (in this case, the distance between the clamps), as shown in Figures 3 and 4 . The rotating speed was set at 12 degree/s. We used the forward torsional rigidity in this paper. The torsional rigidity $W_{\text {mes }}$ during forward rotation is given by Equation (6):

$$
W_{\text {mes }}=\frac{T_{4 \pi}-T_{2 \pi}}{\frac{2 \pi}{L}}
$$

where, $T_{4 \pi}$ and $T_{2 \pi}$ are the torques at $2 \pi$ and $4 \pi$, respectively.
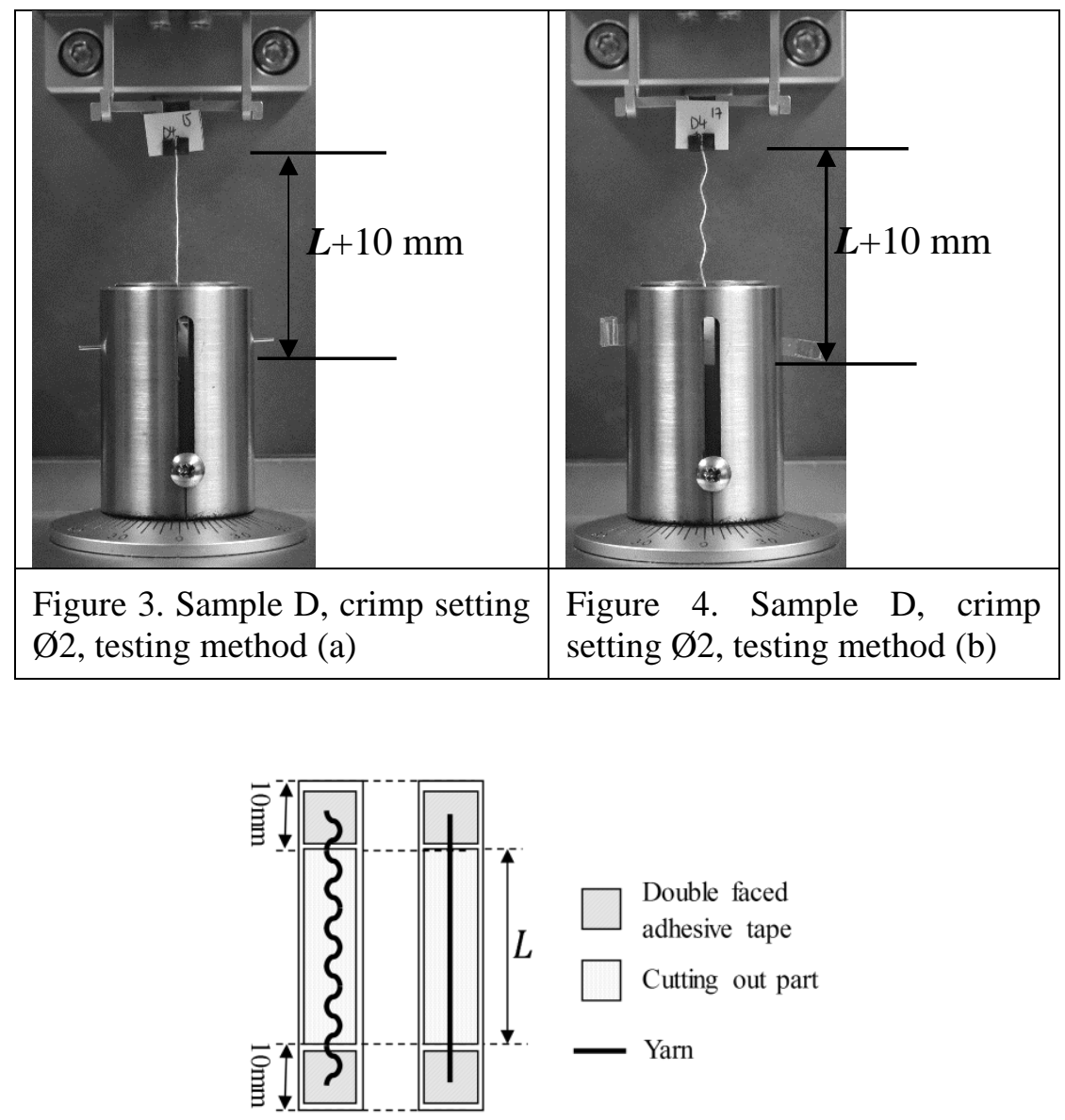

Figure 5. Sample layout with and without crimp, with $L=30 \mathrm{~mm}$

\section{Sample preparation and testing conditions}

Monofilament. The sample length was adapted for every crimp setting so that the test samples had a yarn length of $3 \mathrm{~cm}$. Ten samples of each kind of yarn and each value of crimp, including no-crimp samples, were prepared and tested. The sample layout is shown in Figure 5.

During testing, the monofilament yarn was subjected to a weight of $5.342 \mathrm{~g}$ (clamp +5 -g weight) in addition to its own weight. Due to the weight, the sample length and the distance between the clamps changed. Therefore, the new length was measured for the calculation of rigidity. We attempted to test the samples without the 5-g weight, but the results were inconclusive due to uneven deformation. 
Cotton spun yarn. Cotton spun yarn samples were prepared and tested by the following two methods.

(a) As an initial load, a weight equivalent to $200 \mathrm{~m}$ of yarn was applied when making the sample according to the Japanese Industrial Standard (JIS) for testing fabrics (JIS L1096:2010) [19]. Then, after $24 \mathrm{~h}$ under standard conditions (at $20 \pm$ $1{ }^{\circ} \mathrm{C}$ and a relative humidity of $65 \pm 5 \%$ ), the yarn torsional rigidity was measured. Fifteen samples of each kind of yarn and each crimp setting (including the no-crimp setting) were prepared and tested. During testing, the cotton spun yarn samples were subjected to the weight of the clamp $(0.342 \mathrm{~g})$ in addition to their own weight. An example of a yarn being tested is shown in Figure 4.

(b) No weight was applied during sample production to maintain the crimp. For each crimp setting (including the nocrimp setting), 15 samples were produced. During testing, almost no weight was applied to the samples to preserve the crimp. To reduce the load and maintain the rotation of one end of the sample, a double-sided strip of adhesive tape was used instead of the clamp, as shown in Figure 5. The tape weight was approximately $0.07 \mathrm{~g}$. When put in place, the sample hung down freely under its own weight and that of the tape. When rotating, the adhesive part of the tape maintained the sample in position.

During measurement, a picture was taken of each sample to measure the wavelength $\lambda$ and the length $L$ from the clamp to the end. $L$ was used to calculate the torsional rigidity of the crimped yarns. $S$ was measured after testing by applying a weight to straighten the sample. $L, \lambda$, and $S$ were used to calculate the amplitude $A$, the length $s$ of the yarn for one period $\lambda$, and the crimp ratio $c$.

\section{Results and discussions}

\section{Geometrical parameters in testing}

Table 3 shows the geometrical parameters of the samples during measurement using testing methods (a) and (b), measured from the pictures. The crimp ratio during testing was smaller than that at setting because $\lambda$ increased.

For testing method (a), as the yarn was straightened, $\lambda$ reached a maximum equal to $s$, and consequently $A$ and $c$ were equal to zero. For testing method (b), $A, \lambda, s$, and $c$ decreased due to the weight, but the crimp shapes were still apparent.

Table 3. Crimped yarn geometry during measurements (in $\mathrm{mm}$ )

\begin{tabular}{|l|lllllllllllllll|llll|}
\hline$d_{r}$ & $\emptyset 1.2$ & & & & $\emptyset_{2}$ & & & & $\emptyset 3$ & & & & $\varnothing 4$ & & \\
\hline Samples & $A_{1.2}$ & $\lambda_{1.2}$ & $s_{1.2}$ & $c_{1.2}$ & $A_{2}$ & $\lambda_{2}$ & $s_{2}$ & $c_{2}$ & $A_{3}$ & $\lambda_{3}$ & $s_{3}$ & $c_{3}$ & $A_{4}$ & $\lambda_{4}$ & $s_{4}$ & $c_{4}$ \\
\hline FC & 1.24 & 5.55 & 7.41 & 0.33 & 2.01 & 8.02 & 11.36 & 0.42 & 3.01 & 11.13 & 16.45 & 0.48 & 3.98 & 14.10 & 21.40 & 0.52 \\
Nylon & 1.26 & 5.49 & 7.44 & 0.35 & 2.08 & 7.46 & 11.34 & 0.52 & 3.04 & 11.07 & 16.54 & 0.49 & 3.95 & 14.23 & 21.36 & 0.50 \\
\hline A-a & 0.00 & 7.50 & 7.50 & 0.00 & 0.00 & 11.48 & 11.48 & 0.00 & 0.00 & 16.53 & 16.53 & 0.00 & 0.00 & 21.81 & 21.81 & 0.00 \\
B-a & 0.00 & 7.68 & 7.68 & 0.00 & 0.00 & 11.69 & 11.69 & 0.00 & 0.00 & 16.79 & 16.79 & 0.00 & 0.00 & 22.10 & 22.10 & 0.00 \\
C-a & 0.00 & 7.88 & 7.88 & 0.00 & 0.00 & 11.92 & 11.92 & 0.00 & 0.00 & 17.07 & 17.07 & 0.00 & 0.00 & 22.42 & 22.42 & 0.00 \\
D-a & 0.00 & 8.09 & 8.09 & 0.00 & 0.00 & 12.16 & 12.16 & 0.00 & 0.00 & 17.36 & 17.36 & 0.00 & 0.00 & 22.75 & 22.75 & 0.00 \\
\hline A-b & 1.24 & 5.29 & 7.26 & 0.37 & 2.00 & 7.51 & 11.04 & 0.47 & 2.96 & 10.53 & 16.00 & 0.52 & 3.99 & 13.78 & 21.33 & 0.55 \\
B-b & 1.26 & 5.38 & 7.39 & 0.37 & 2.06 & 7.52 & 11.33 & 0.51 & 3.03 & 10.20 & 16.17 & 0.59 & 4.10 & 12.89 & 21.55 & 0.67 \\
C-b & 1.25 & 5.31 & 7.37 & 0.39 & 2.07 & 7.54 & 11.39 & 0.51 & 3.13 & 10.34 & 16.72 & 0.62 & 3.93 & 12.15 & 20.71 & 0.70 \\
D-b & 1.26 & 5.23 & 7.38 & 0.41 & 2.02 & 7.46 & 11.24 & 0.51 & 2.90 & 10.13 & 15.74 & 0.55 & 3.98 & 12.79 & 21.13 & 0.65 \\
\hline
\end{tabular}

\section{Typical torque curves for monofilament yarn and cotton spun yarn}

Figure 6 shows an example of a typical torque curve for monofilament yarn. Because FC and Nylon monofilaments are homogeneous materials, the samples produced the same response in both the forward and backward direction, giving a symmetrical curve. For the FC monofilaments, the crimp setting decreased the torsional rigidity of the samples compared with the straight samples. For the Nylon monofilaments, the opposite was true: the torsional rigidity of the straight yarn was lower than that of the crimped yarn.

Figure 7 shows an example of a typical torque curve for the cotton spun yarn. The torsional rigidity and hysteresis in the forward direction were larger than in the backward direction. This was due to the twisted structure of the cotton spun yarn. For the cotton spun yarn, the torsional rigidity of the yarn without crimp was larger than that of the crimped yarn. 
Furthermore, the results of applying a weight during testing (method a) showed a similar torsional rigidity for the yarns preserving crimp (method b).

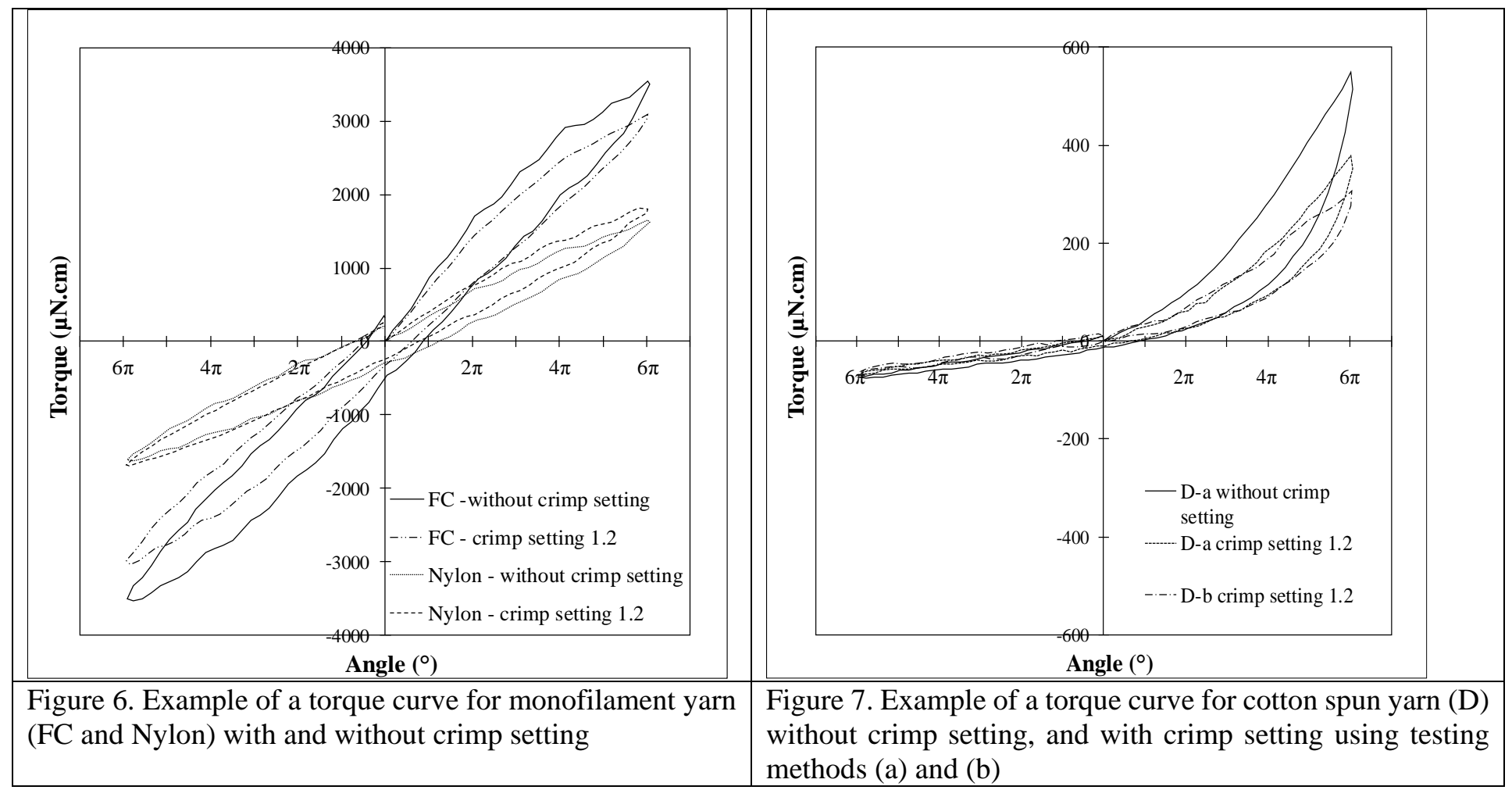

\section{Relationship between torsional rigidity and crimp setting of monofilament yarns}

Figure 8 shows the torsional rigidities of FC and Nylon monofilament yarns for each crimp setting. The torsional rigidity of the FC monofilaments decreased as the crimp setting increased. The Nylon monofilament torsional rigidity, in contrast, did not show any variation with the different crimp settings. According to Equation (4), the torsional rigidity ratio $W / W_{2}$ increase or decrease with crimp depend on $W / B$ [17]. $W / B$ depends on Poisson ratio in the case of isotropic materials. The difference is caused by their different Poisson's ratio. For the monofilament yarns, few tests were necessary because the results were very consistent and had a very low standard deviation.

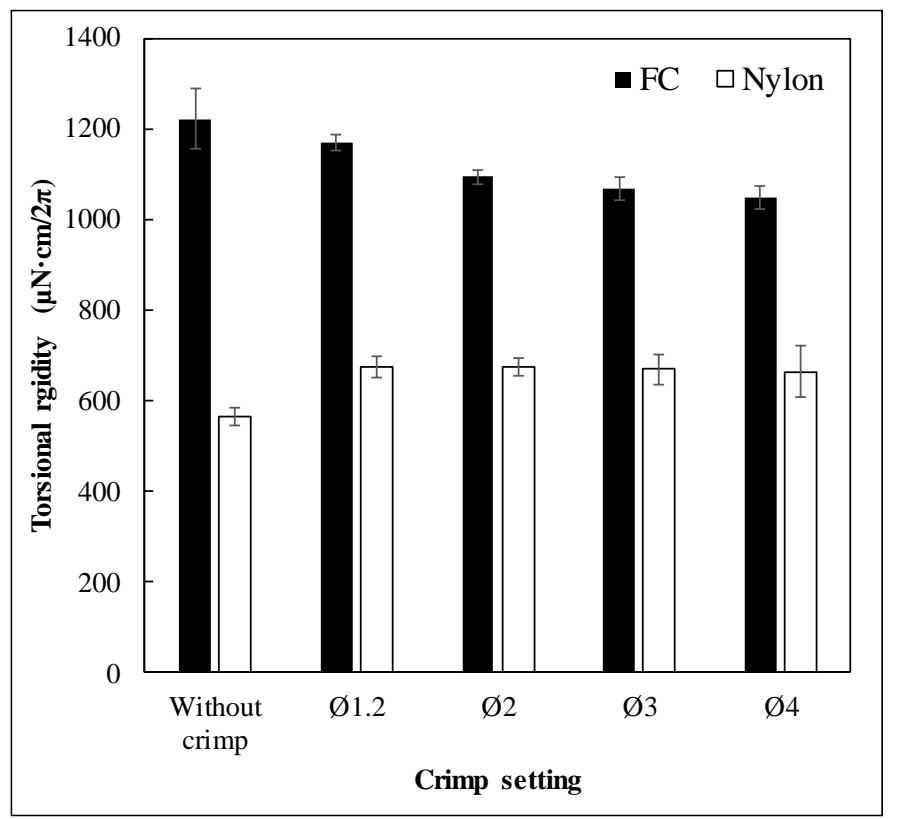

Figure 8. Torsional rigidity of FC and Nylon monofilament yarns for each yarn setting 


\section{Relationship between torsional rigidity and crimp setting of cotton yarn}

Effect of the crimp setting Figures 9 to 12 show the torsional rigidity of the cotton spun yarn samples (A to D) for each crimp setting. For all samples, the torsional rigidities of the yarns without crimp were greater than those with crimp. For the samples with crimp settings, the smaller the crimp, the lower the crimp rigidity. There was generally a small difference between samples with (a) and without (b) the application of a weight during crimp setting, and both testing methods showed almost similar torsional rigidities.

This means that the torsional rigidity of the crimped yarn was the same whether or not it had been straightened at the time of the test. This could have been due to the fibers being bent when the crimp was set on the yarn, and staying bent even when the yarn was straightened.

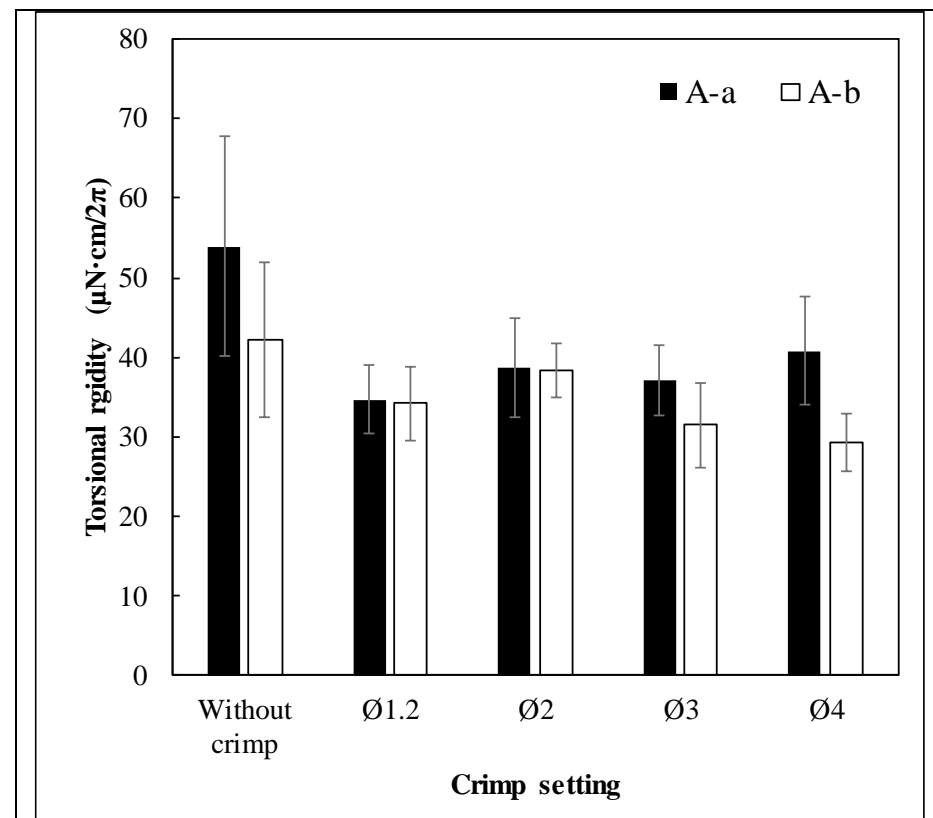

Figure 9. Torsional rigidities of $\mathrm{A}$ with (A-a) and without (A-b) weight

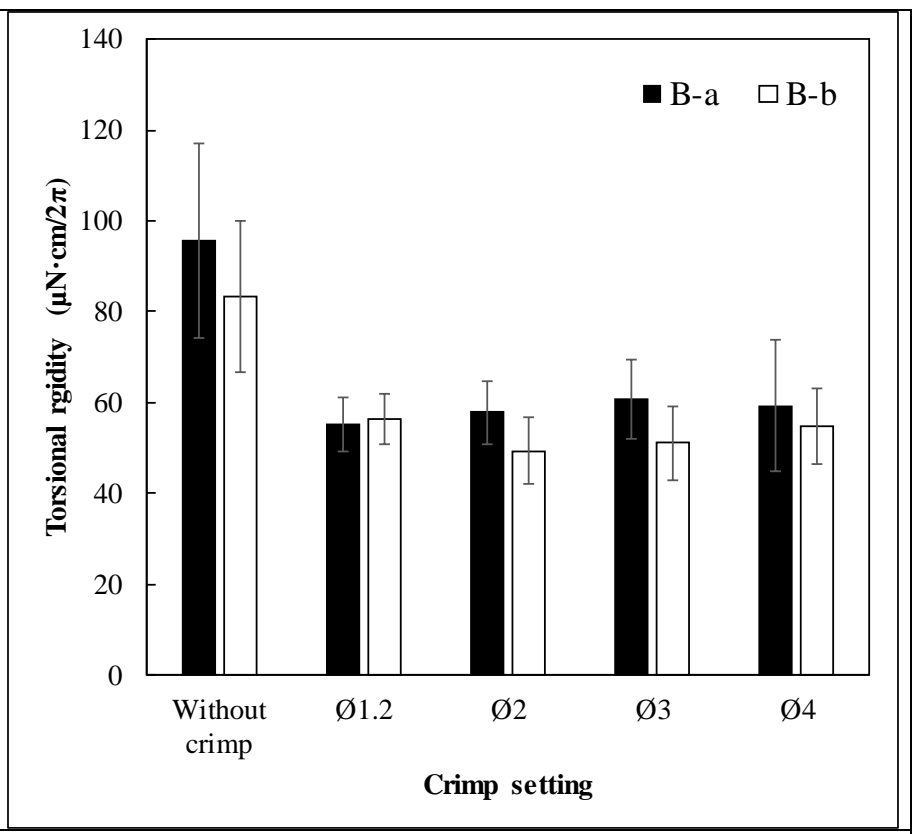

Figure 10. Torsional rigidities of $\mathrm{B}$ with (B-a) and without (B-b) weight

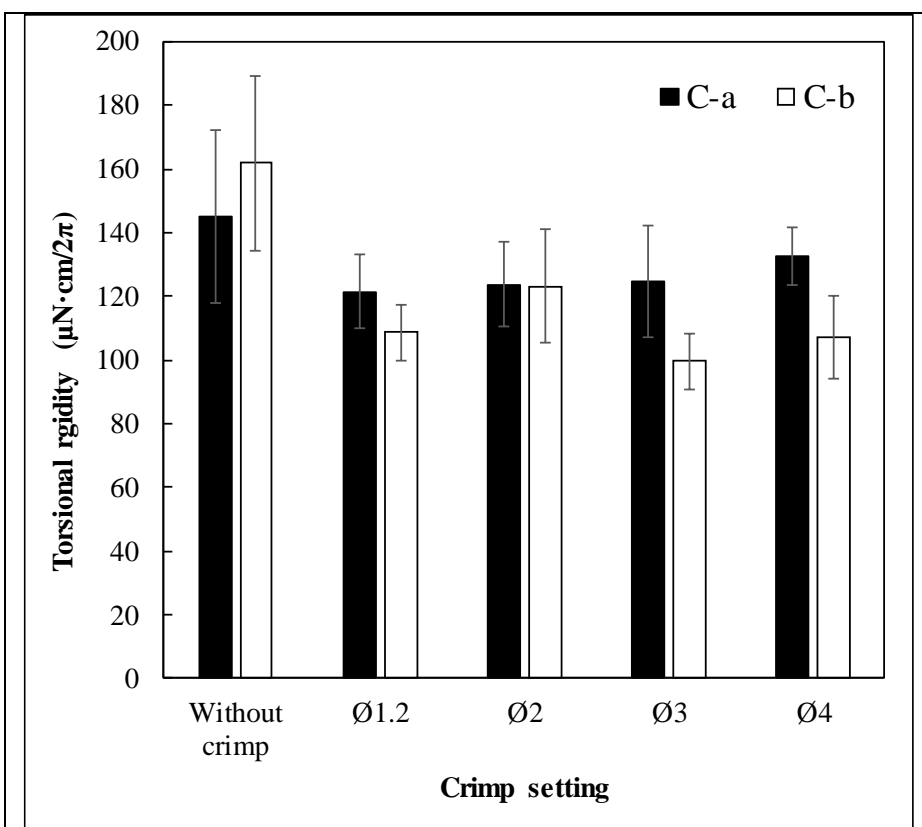

Figure 11. Torsional rigidities of $\mathrm{D}$ with (D-a) and without (D-b) weight

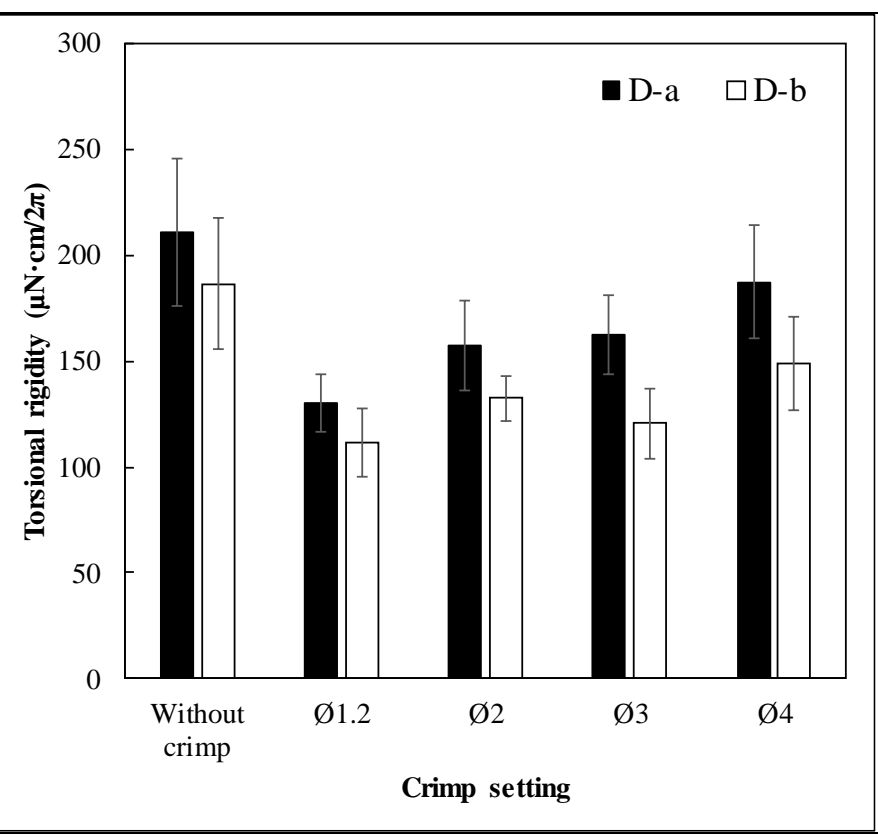

Figure 12. Torsional rigidities of $\mathrm{B}$ with (D-a) and without (D-b) weight 
Effect of the yarn count Figures 13 and 14 show the results for all samples (A to D) for testing methods (a) and (b). As expected, the smaller the yarn count, the smaller the yarn torsional rigidity. Therefore, we found that the effect of crimp on torsional rigidity differed according to the yarn count.

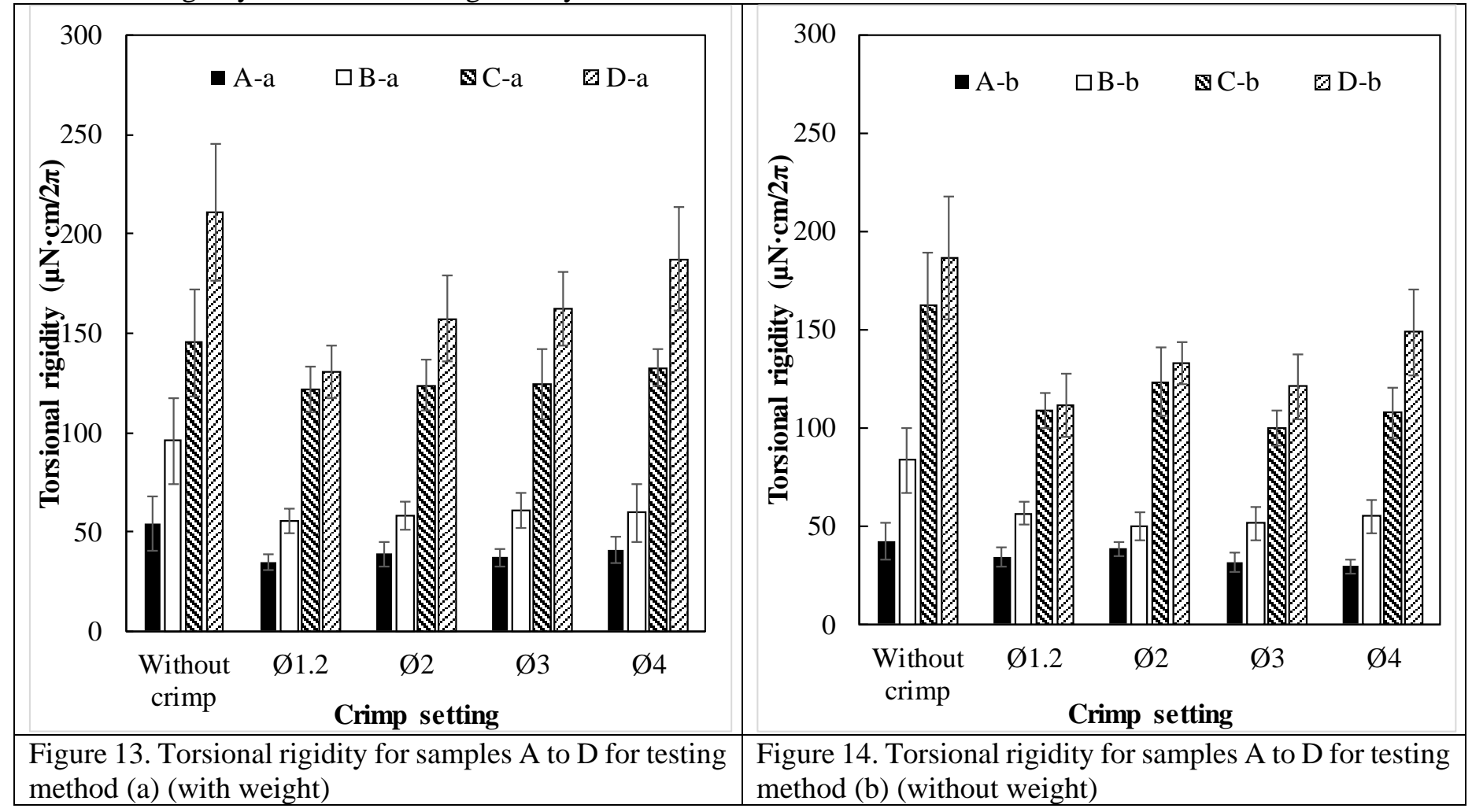

\section{Relationship between the torsional rigidity ratio and the crimp ratio}

Figure 15 shows the relationship between the torsional rigidity ratio $W / W_{2}$ (where $W$ is the torsional rigidity of the yarn without crimp and $W_{2}$ is the torsional rigidity of the crimped yarn) and the crimp ratio $c$ (Equation (5)). For the monofilament yarns, $W / W_{2}$ had an almost linear relationship with the crimp ratio. The $W_{2}$ of the FC monofilament was smaller than $W$, and $W / W_{2}$ increased with the crimp ratio. Conversely, the $W_{2}$ of the Nylon monofilament was larger than the $W$, and $W / W_{2}$ decreased slightly in the tested crimp range. This could have been due to the bending rigidity $B$, as shown in Equation (4). The results for the cotton spun yarns showed the large effect of the crimp. The $W_{2}$ values for the cotton spun yarns were smaller than the $W$ values. According to Equation (4), the torsional rigidity ratio and the crimp ratio didn't present a monotonic increase for smaller W/B [17]. The results in Figure 15 include this phenomenon. However, no clear relationship was observed due to the high variance in the torsional rigidity results, as shown in Figures 9 to 12. However, it can be noted that the torsional rigidity ratio of cotton spun yarn samples were higher than the ones of monofilaments. 


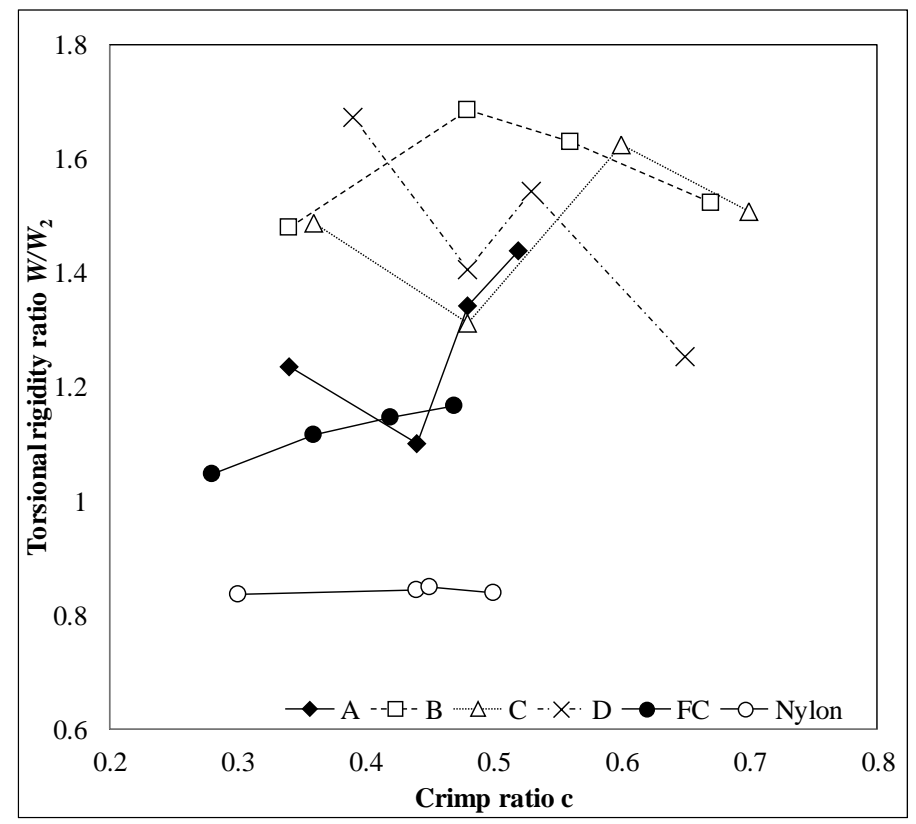

Figure 15. Relationship between torsional rigidity ratio and crimp ratio

\section{Conclusion}

In this study, the effect of crimp on the torsional rigidity of monofilament yarns and cotton spun yarns was investigated. Crimped monofilaments and spun yarns with various amplitudes and wavelengths were produced using original crimp setting equipment. The torque-twist curves of the straight and crimped yarns were measured using a torsional tester, and their torsional rigidities were obtained.

The results showed that the torsional rigidities of all the straight yarns except the nylon monofilament were higher than those of the crimped yarns. For the cotton spun yarn, the torsional rigidity of crimped yarn was smaller than the one of the straight yarn. The variation of torsional rigidity in between samples with the same yarn count for different crimp setting wasn't however significant. As expected, the smaller the yarn count, the smaller the torsional rigidity. It was also noted that straightening after crimp setting made almost no difference to the torsional rigidity of the crimped yarn. Therefore, there is a possibility that the property changes in the yarn were due to the fiber being bent during crimp setting, and were not due to the shape of the crimp afterwards.

After calculating the ratio between torsional rigidity of straight and crimped yarn, the relationship between the torsional rigidity ratio and the crimp ratio was studied. The monofilament results showed nearly linear relationships between the torsional rigidity ratio and the crimp ratio. On the other hand, the torsional rigidity ratio for cotton spun yarn did not show a clear relationship with the crimp ratio due to a higher variance in the testing results. However, it can be noted that the torsional rigidity ratio of cotton spun yarn samples were higher than the ones of monofilaments.

This study demonstrated the effect of crimp on the torsional rigidity of yarn, which had not been clarified experimentally until now.

\section{Acknowledgements}

This work was supported by JSPS KAKENHI under grant numbers JP24220012.

\section{References}

1. Inui $\mathrm{S}$, Okabe $\mathrm{H}$, Takatera $\mathrm{M}$ et al. A fabric simulation based on a model constructed from a lower level. In: Computational Engineering in Systems Applications, IMACS Multiconference on, Volume 1. Beijing: IEEE, 2006, pp.178-180.

2. Inui S, Mesuda Y, Horiba Y et al. Fabric weave form simulation based on the characteristics of yarn. Biometrics and Kansei Engineering (ICBAKE), 2011 International Conference on. Takamatsu, Kagawa: IEEE, 2011, pp.49-52.

3. Kawabata S, Niwa M and Kawai H. 3-The finite-deformation theory of plain-weave fabrics part I: The biaxialdeformation theory. J Tex Inst 1973; 64: 21-46.

4. Cooper DNE. 24-The stiffness of woven textiles. J Tex Inst 1960; 51: T317-T335.

5. Platt MM, Klein WG and Hamburger WJ. Mechanics of elastic performance of textile materials: Part XIII: Torque development in yarn system: Singles yarn. Tex Res J 1958; 28: 1-14. 
6. Brand RH and Backer S. Mechanical principles of natural crimp of fiber. Tex Res J 1962; 32: 39-49.

7. Menkart J and Detenbeck JC. The significance of wool fiber crimp part I: A study on the worsted system. Tex Res $J$ 1957; 27: 665-689.

8. Horio M and Kondo T. Crimping of wool fibers. Tex Res J 1953; 23: 373-386.

9. Meredith R. 30 - The torsional rigidity of textile fibres. J Tex Inst 1954; 45: T489-T503.

10. Brand RH and Scruby RE. Three-dimensional geometry of crimp. Tex Res J 1973; 43: 544-555.

11. Peirce FT. 26-The "handle" of cloth as a measurable quantity. J Tex Inst 1930; 21: T377-T416.

12. Olofsson B. 49-A general model of a fabric as a geometric mechanical structure. J Tex Inst 1964; 55: T541-T557.

13. Postle R, Burton P and Chaikin M. 41-The torque in twisted singles yarns. J Tex Inst 1964 ; 55: T448-T461.

14. Dhingra RC and Postle R. 19-The measurement of torque in continuous-filament yarn. Part II: The effect of yarn tension. J Tex Inst 1974; 65: 171-181.

15. Shinohara F, Shinohara A and Sakaebara K. A theoretical study on the anisotropy of the bending rigidity of woven fabrics. J Textil Mach Soc Jpn 1979; 32: T66-T71.

16. Peiffer J, Kim K and Takatera M. Verification of the effect of yarn torsional rigidity on fabric bending rigidity in any direction. Tex Res J 2016; doi: 10.1177/0040517516631321.

17. Skelton J. 38 - The effect of planar crimp in the measurement of the mechanical properties of fibres, filaments, and yarns. J Tex Inst 1967; 58: 533-556.

18. Takatera M, Arichi T, Peiffer J, Zhu C et al. Continuous measurement of apparent Poisson's ratio for yarn based on omni-directional diameters. Tex Res J 2016; doi: 10.1177/0040517516639817.

19. JIS. Handbook 54 Sen 'i. Japan: Japanese Standards Association, 2010. 